\title{
ULAS J141623.94+134836.3 - a faint common proper motion companion of a nearby $L$ dwarf
}

\section{Serendipitous discovery of a cool brown dwarf in UKIDSS DR6}

\author{
R.-D. Scholz
}

\author{
Astrophysikalisches Institut Potsdam, An der Sternwarte 16, 14482 Potsdam, Germany \\ e-mail: rdscholz@aip.de
}

Received 15 January 2010 / Accepted 27 January 2010

\section{ABSTRACT}

\begin{abstract}
Aims. New near-infrared large-area sky surveys (e.g. UKIDSS, CFBDS, WISE) go deeper than 2MASS and aim at detecting brown dwarfs lurking in the solar neighbourhood that are even fainter than the latest known T-type objects, so-called Y dwarfs.

Methods. Using UKIDSS data, we found a faint brown dwarf candidate with very red optical-to-near-infrared, but extremely blue near-infrared colours next to the recently discovered nearby L dwarf SDSS J141624.08+134826.7. We checked if the two objects are co-moving by studying their parallactic and proper motion and compared the new object with known T dwarfs.

Results. The astrometric measurements are consistent with a physical pair (sep $\approx 75 \mathrm{AU})$ at a distance $d \approx 8$ pc. The extreme colour $(J-K \approx-1.7)$ and absolute magnitude $\left(M_{J}=17.78 \pm 0.46\right.$ and $\left.M_{K}=19.45 \pm 0.52\right)$ make the new object appear as one of the coolest $\left(T_{\text {eff }} \approx 600 \mathrm{~K}\right)$ and nearest brown dwarfs, probably of late-T spectral type and possibly with a high surface gravity $(\log g \approx 5.0$ ).
\end{abstract}

Key words. astrometry - stars: distances - stars: kinematics and dynamics - brown dwarfs - solar neighborhood

\section{Introduction}

One of the open questions of low-mass star formation is the ratio of successful/failed star formation processes. In other words, is the solar neighbourhood populated by as many cool brown dwarfs as red dwarf stars? Several new near-infrared surveys like UKIDSS ${ }^{1}$, CFBDS (Delorme et al. 2008b) and WISE (Wright 2008; Mainzer et al. 2009) try to answer this question by going deeper than the Two Micron All Sky Survey (2MASS; Skrutskie et al. 2006) to detect a new class of ultracool brown dwarfs, so-called Y dwarfs.

The appearance of ammonia absorption in the near-infrared spectra is being discussed as a criterion for the new $\mathrm{Y}$ spectral type (Burningham et al. 2008; Delorme et al.2008b). Whereas the latest-type (coolest) objects discovered in the 2MASS are of spectral type T8 (Burgasser et al.2002; Tinney et al. 2005; Looper et al. 2007), a handful of even cooler ( $\left.T_{\text {eff }} \approx 500-600 \mathrm{~K}\right)$ brown dwarfs (T8.5-T9) have already been discovered in UKIDSS (Warren et al. 2007; Burningham et al. 2008, 2009) and CFBDS (Delorme et al. 2008a) that do not look conspicuously different in their near-infrared spectra from latetype T dwarfs. A unique $\mathrm{Y}$ dwarf has not yet been found and classified.

We describe a new cool brown dwarf, which is probably a late-T dwarf with unusual properties, detected as a wide companion of a nearby blue L dwarf.

1 The UKIDSS project is defined in Lawrence et al. (2007). UKIDSS uses the UKIRT Wide Field Camera (WFCAM; Casali et al. 2007) and a photometric system described in Hewett et al. (2006) which is situated in the Mauna Kea Observatories (MKO) system (Tokunaga et al. 2002). The pipeline processing and science archive are described in Irwin et al. (2010, in prep.) and Hambly et al. (2008).

\section{Identification of a faint object with unusual colors near the blue L dwarf SDSS J141624.08+134826.7}

While inspecting the UKIDSS finding charts around the recently discovered (Schmidt et al. 2010, hereafter S10; Bowler et al. 2010, hereafter B10) nearby blue L6 dwarf SDSS J141624.08+134826.7 (hereafter called object A), we found a fainter nearest neigbouring object with extreme colours, separated by about 9.4 arcsec. This object, ULAS J141623.94+134836.3 (hereafter called object B) was not detected in the SDSS DR7 (Abazajian et al. 2009), but is well measured in the UKIDSS, where it has $Y-J \approx+0.9$, but a very blue near-infrared colour of $J-H \approx-0.3$ and $J-K \approx-1.7$ (Fig. 1; Table 1). Abazajian et al. (2009) describe the completeness limit of SDSS DR7 with a 95\% detection repeatability for point sources at $u=22.0, g=22.2, r=22.2, i=21.3$, and $z=20.5$. The non-detection of object B in SDSS DR7 hints at a very red optical-to-near-infrared colour $(z-Y>+2.3$ and $z-J>+3.1)$. Using two available overlapping $z$-band FITS images (SDSS runs 3971 and 3996) downloaded from the SDSS DR7, we were able to detect object B (for the astrometry see Table 2) and measure its magnitude as $z_{3971}=21.24 \pm 0.50$ and $z_{3996}=21.02 \pm 0.39$. The resulting mean colour indices are $z-Y=+2.97 \pm 0.32$ and $z-J=+3.87 \pm 0.32$.

Comparing the near-infrared colour indices of object B with those of the known T dwarfs (Fig. 2), one can see that similar moderately negative $J-H$ have been measured for the latest-type (T9), but also for other mid- and late-type T dwarfs, whereas the extremely large negative $J-K$ of object B clearly stands out against the rest of the T dwarfs. Both colours are in the range typical of model $\mathrm{T}$ and $\mathrm{Y}$ dwarfs, but rule out a highredshift quasar (Hewett et al. 2006). However, before further 
Table 1. SDSS DR7 $z$ (on the AB system) and UKIDSS DR6 YJHK (on the Vega system using the MKO photometric system) photometry.

\begin{tabular}{lccccc}
\hline \hline object & mean $z$ & $Y$ & $J$ & $K$ & \\
& $(3971+3996)$ & $(2325240)$ & $(2325252)$ & $(1906043)$ & $(1905923)$ \\
\hline SDSS J141624.08+134826.7 (object A) & $15.897 \pm 0.005$ & $14.255 \pm 0.003$ & $12.995 \pm 0.001$ & $12.469 \pm 0.001$ & $12.053 \pm 0.001$ \\
ULAS J141623.94+134836.3 (object B) & $21.13 \pm 0.32^{*}$ & $18.162 \pm 0.027$ & $17.259 \pm 0.017$ & $17.581 \pm 0.030$ & $18.933 \pm 0.244$ \\
\hline
\end{tabular}

Notes. ${ }^{*}$ Not detected in SDSS DR7 (see text). $z$ magnitudes are mean values from two SDSS runs (given in brackets). $Y J H K$ magnitudes are aperMag3 derived from the multiframes (given in brackets) for point sources (Dye et al. 2006). A second set of $Y J H K$ measurements was not used due to the location of objects A and B close to the edge of the frames.

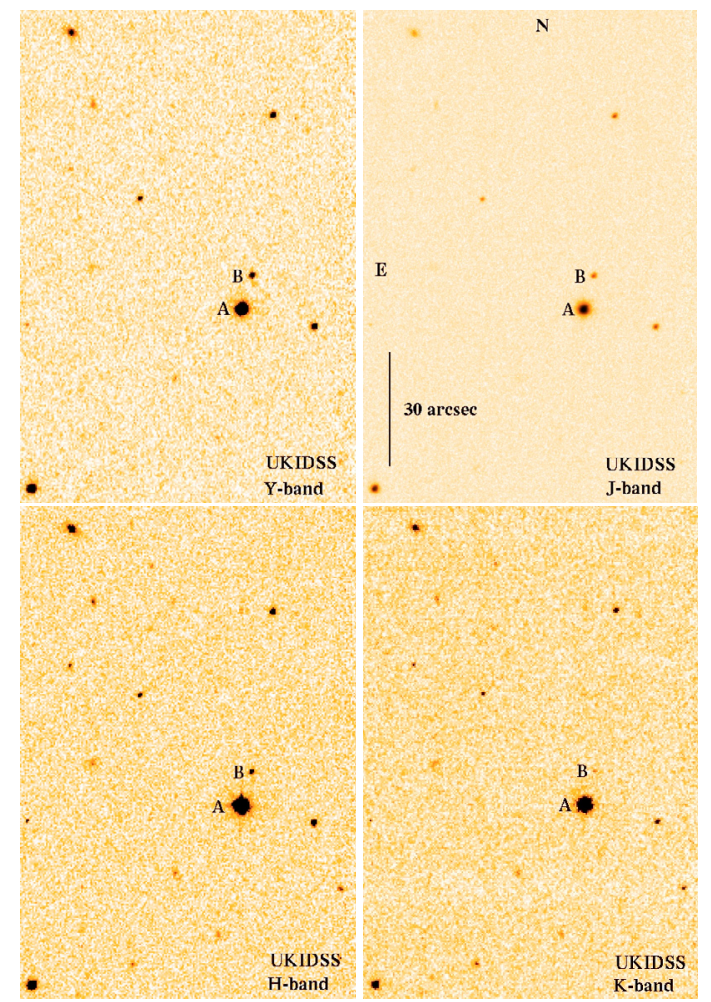

Fig. 1. UKIDSS $Y J H K$ images of objects A and B. Image scale and orientation (North is up, East to the left) are shown in the $J$-band image.

analysis we need to check the physical association of object B with object $\mathrm{A}$ and to confirm its distance.

\section{Confirmation of common proper motion}

For a first check of a possible common motion of objects A and $\mathrm{B}$, one can use the accurate UKIDSS data alone. There are two different epochs for the $H K$ and $Y J$ observations respectively (Table 2). The corresponding multiframe numbers are listed in Table 1. Short-term proper motions were determined from simple linear fitting over the four epoch positions of objects $\mathrm{A}$ and $\mathrm{B}$ as well as of six field stars in their vicinity, well measured on the same multiframes (Fig. 3). Significant results, which agree within their errors, were obtained for A and B (solution 1-A and 1-B in Table 3). The common short-term proper motion of $\mathrm{A}$ and $\mathrm{B}$ is a first strong hint on a physical pair, but it is more spread out and in a different direction than the longterm proper motions of object A obtained by S10 and B10. We will show that this discrepancy can be explained by the expected common parallactic motion.

With two $z$-band SDSS images that contain object A, we were able to detect object B with the help of the ESO skycat

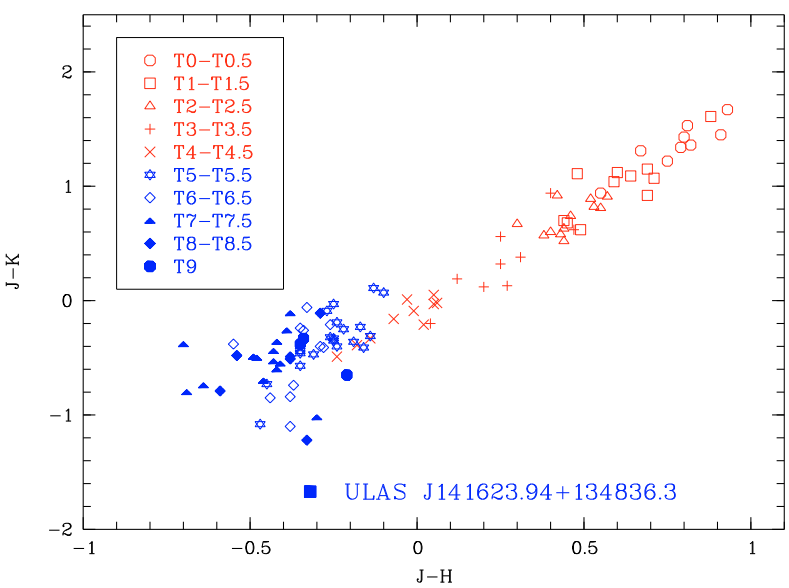

Fig. 2. Near-infrared two-colour diagram $J-K$ vs. $J-H$ (in MKO system) for all T dwarfs in Leggett et al. (2010) and the new cool brown dwarf candidate ULAS J141623.94+134836.3 (object B).

Table 2. Multi-epoch positions $\alpha, \delta$ (J2000.0): $14^{\mathrm{h}} 16^{\mathrm{m}} \ldots{ }^{\mathrm{s}},+13^{\circ} 48^{\prime} \ldots{ }^{\prime \prime}$

\begin{tabular}{lcccll}
\hline \hline \multicolumn{1}{c}{ s } & \multicolumn{1}{c}{ s } & s & \multicolumn{1}{c}{$\begin{array}{l}\text { Epoch } \\
\text { yr }\end{array}$} & Source \\
\hline 23.804 & 19.88 & & & 1954.338 & SSS $E$ \\
24.050 & 25.48 & & & 1994.338 & SSS $R$ \\
24.068 & 25.85 & & & 1997.521 & SSS $I$ \\
24.0847 & 26.345 & & & 2000.164 & 2MASS \\
24.0886 & 26.741 & $23.886^{*}$ & $35.88^{*}$ & 2003.409 & SDSS 3971 \\
24.0859 & 26.683 & $23.916^{*}$ & $35.49^{*}$ & 2003.472 & SDSS 3996 \\
24.1305 & 27.339 & 23.9480 & 36.268 & 2008.189 & UKIDSS $H$ \\
24.1325 & 27.355 & 23.9466 & 36.273 & 2008.189 & UKIDSS $K$ \\
24.1260 & 27.410 & 23.9421 & 36.319 & 2008.362 & UKIDSS $Y$ \\
24.1254 & 27.416 & 23.9435 & 36.334 & 2008.362 & UKIDSS $J$ \\
\hline
\end{tabular}

Notes. ${ }^{(*)}$ Not detected in SDSS DR7 (see text).

tool and the "pick object" option that is based on Gaussian fitting (Table 2). We think the reason why object B does not appear in the SDSS DR7 is that it is $>0.5 \mathrm{mag}$ fainter than the already mentioned $95 \%$ detection limit in $z$ and cannot be detected in ugri, where it should be much fainter than the corresponding limits. Using now our two SDSS positions of object B together with its four UKIDSS positions, we again got similar proper motions (solutions 2-A and 2-B in Table 3), now also approaching the known long-term proper motion of object $\mathrm{A}$. We further improved this (Fig. 4, solution 3-A in Table 3) by including all available epochs, also 2MASS and SuperCOSMOS Sky Surveys (SSS; Hambly et al. 2001) data (Table 2). Note that S10 did not use UKIDSS, whereas B10 missed the important old SSS $E$ epoch for their proper motion solutions of object A.

The spectrophotometric distance of object A is still very uncertain $(8.0 \pm 1.6 \mathrm{pc}$ according to $\mathrm{S} 10$ and $8.4 \pm 1.9 \mathrm{pc}$ according 
R.-D. Scholz: ULAS J141623.94+134836.3 - a faint common proper motion companion of a nearby L dwarf

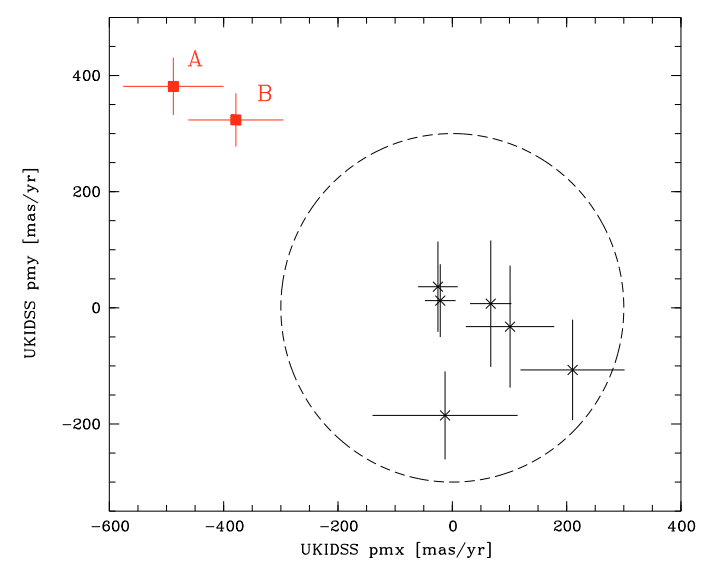

Fig. 3. Short-term proper motions (from $2 \times 2$ UKIDSS epochs separated by 0.17 years) of objects $A$ and $B$ (filled squares, solutions 1-A and 1-B in Table 3) in comparison to those of field stars (crosses). Typical proper motion errors of $\sigma \approx 75 \mathrm{mas} / \mathrm{yr}$ were achieved in both directions. The dashed circle represents a $4 \sigma$ significance level.

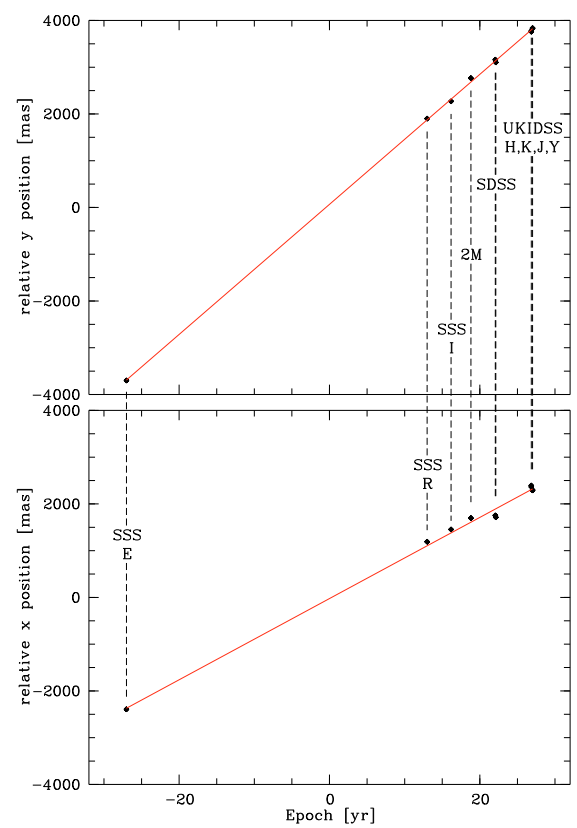

Fig. 4. Linear proper motion fit for object A (solution 3-A).

to B10), because the spectral type-absolute magnitude relations are not yet well determined for the class of blue $\mathrm{L}$ dwarfs. B10 mentioned a notable parallactic motion of object A, but their trigonometric parallax $\left(\pi_{\text {rel }}=107 \pm 34\right.$ mas $)$ leads to a less accurate distance $(9.3 \pm 3.0 \mathrm{pc})$ than the aforementioned spectrophotometric distance estimates.

We applied the software of Gudehus (2001) for combined proper motion and parallax solutions. In the full solution for object A (solution 4-A), we made use of all ten available epochs, assigning the following uncertainties to the $\alpha, \delta$ given in Table 2: 70 mas for UKIDSS and SDSS, 100 mas for 2MASS, 150 mas for SSS $I$-band, 200 mas for SSS $R$-band, and 250 mas for the SSS measurement of the old $E$ plate (expected colour-dependent systematic errors in the different $\alpha, \delta$ are much smaller and have been neglected). As an alternative, we used only the most accurate data (UKIDSS and SDSS) and the proper motion obtained in solution 3-A as a fixed input parameter in fitting only the parallactic motion of object A (solution 5-A in Table 3 ) and object B
Table 3. Proper motion and parallax solutions for objects A and B.

\begin{tabular}{crrr}
\hline \hline Solution & $\begin{array}{r}\mu_{\alpha} \cos \delta \\
\mathrm{mas} / \mathrm{yr}\end{array}$ & $\begin{array}{r}\mu_{\delta} \\
\mathrm{mas} / \mathrm{yr}\end{array}$ & $\begin{array}{r}\pi_{\text {rel }} \\
\text { mas }\end{array}$ \\
\hline 1-A & $-488.1 \pm 87.8$ & $+381.3 \pm 49.4$ & \\
1-B & $-378.6 \pm 83.3$ & $+323.5 \pm 45.7$ & \\
2-A & $+123.9 \pm 10.1$ & $+132.7 \pm 28.3$ & \\
2-B & $+138.3 \pm 05.8$ & $+126.7 \pm 25.4$ & \\
3-A & $+86.8 \pm 02.1$ & $+139.1 \pm 00.9$ & \\
4-A & $+\mathbf{8 6 . 2} \pm \mathbf{0 2 . 6}$ & $+\mathbf{1 3 8 . 8} \pm \mathbf{0 2 . 6}$ & $\mathbf{1 2 7 . 0} \pm \mathbf{2 7 . 0}$ \\
5-A & & & $153.7 \pm 20.5$ \\
5-B & & & $104.4 \pm 45.5$ \\
\hline
\end{tabular}

Notes. Linear fit using UKIDSS (1), UKIDSS+SDSS (2), all data for A (3). Combined proper motion and parallax solution for A (4). Parallax solution using UKIDSS+SDSS and the previously determined linear proper motion of object $A$ as input (5).

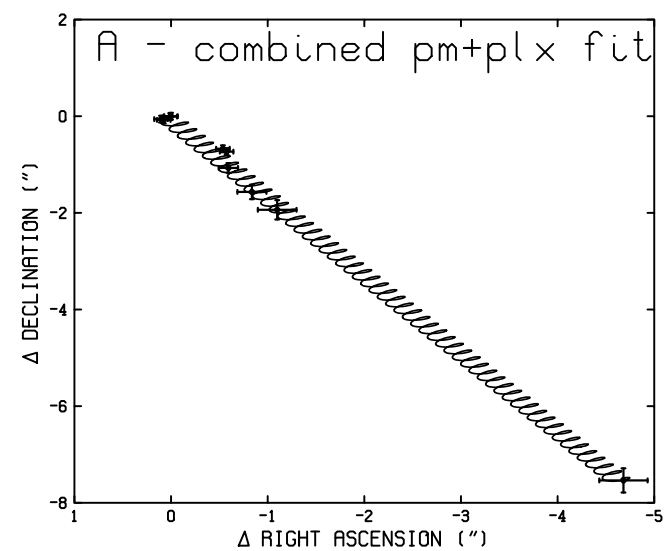

Fig. 5. Combined proper motion and parallax solution with all available epochs for object A (solution 4-A). Zero point is the UKIDSS $J$ epoch.

(solution 5-B). In the latter case we assigned uncertainties of 200 mas to our SDSS $\alpha, \delta$ measurements.

Our preferred solution for object A (4-A) gives a proper motion nearly identical to the linear fit (3-A) and provides a parallax leading to a distance of $7.9 \pm 1.7 \mathrm{pc}$ in perfect/good agreement with the spectrophotometric distances of S10/B10, respectively. Its accuracy is also comparable with that of the spectrophotometric estimates. However, the full range of the parallaxes \pm errors in solutions 4-A, 5-A, and 5-B implies a larger uncertainty for the system. Figure 6 shows that the short-term (UKIDSS only) proper motion of both objects (solutions 1-A and 1-B) is well explained by their common parallactic motion (the parallax results of solutions 5-A and 5-B agree within their errors).

\section{Conclusions and discussion}

We discovered a faint common proper motion companion (object B) of a blue nearby L6 dwarf (object A). Based on the astrometric measurements, which are consistent with a wide binary (projected physical separation $75 \mathrm{AU}$ ) at a distance of about $8 \mathrm{pc}$, and on the accurate near-infrared colours placing object B at the end of the T dwarf sequence (Figs. 2, 7), we conclude that this object is one of the coolest known brown dwarfs, probably with a late-T spectral type.

The latest-type brown dwarfs with trigonometric parallaxes available are the T8.5 dwarfs Wolf 940B at a distance of $12.5 \pm 0.7$ pc (=ULAS J214638.83-001038.7; Burningham et al. 2009; with a parallax measurement for the primary Wolf 940A by Harrington \& Dahn 1980) and 

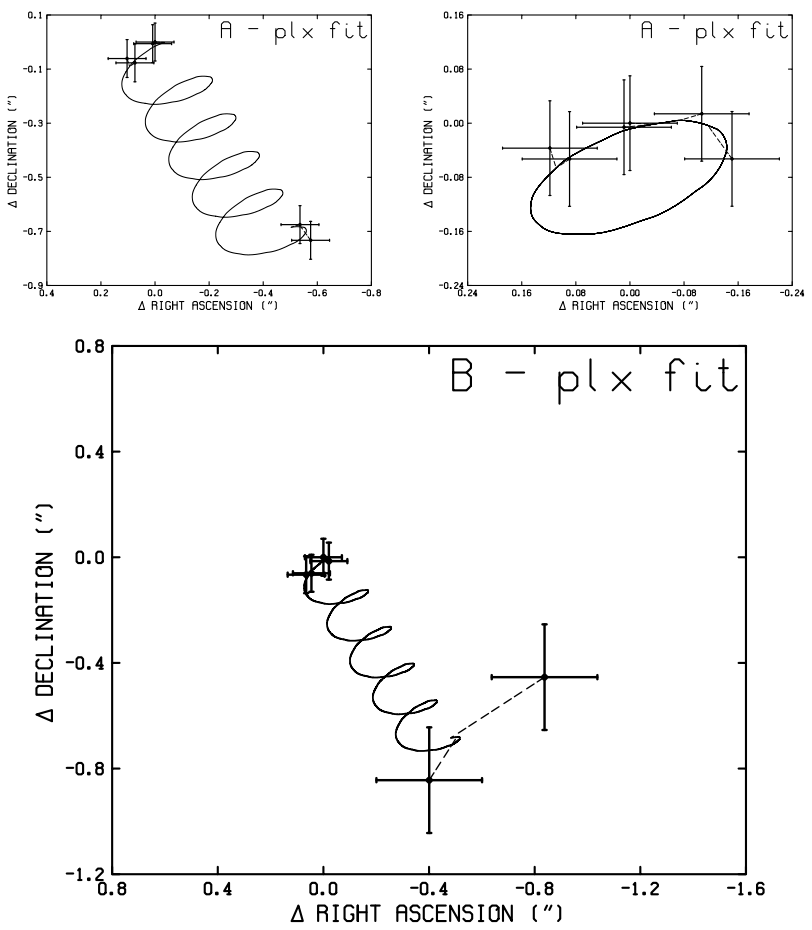

Fig. 6. Parallax solution for objects A (top, solution 5-A; top, right shows the parallax fit with the proper motion removed) and B (bottom, solution 5-B) using UKIDSS+SDSS and the known long-term proper motion (from solution 3-A) as fixed input parameter.

ULAS J003402.77-005206.7 (Warren et al. 2007; Smart et al. 2009) at a distance of $12.6 \pm 0.6 \mathrm{pc}$. Gelino et al. (2009) list only one more T8.5 (ULAS J123828.51+095351.3; Burningham et al. 2008) and two T9 dwarfs (ULAS J133553.45+113005.2; Burningham et al. 2008 and CFBDS J005910.90-011401.3; Delorme et al. 2008a), which are still lacking trigonometric parallaxes. However, their spectrophotometric estimates hint at distances of (slightly) more than $8 \mathrm{pc}$. Object B is by $0.5-1.7 \mathrm{mag}$ brighter in the $J$ - and $H$-band than the above mentioned five objects. In particular, the possibly nearest of the objects, the T9 dwarf ULAS J133553.45+113005.2 at 8-12 pc according to Burningham et al. (2008) is 0.5-0.6 mag fainter than object B in the $Y J H$-bands, whereas it is about 0.5 mag brighter than object $\mathrm{B}$ in the $K$-band. Adopting the mean distance of $10 \mathrm{pc}$ for ULAS J133553.45+113005.2 and 8 pc for object B, their absolute $Y J H$ magnitudes are comparable, whereas object $\mathrm{B}$ is fainter in $M_{K}$ (Fig. 7). Therefore we think that object B is probably the nearest among the latest-type brown dwarfs and offers excellent opportunities for follow-up observations.

With an $H-K \approx-1.35$ and $M_{H} \approx 18.1$, object B falls outside Fig. 9 (top panel) in Leggett et al. (2010), where these authors compare T dwarf observations with models. However, extrapolating the model line with solar metallicity but high gravity $(\log g=5.0)$ gives the best fit, possibly with a $T_{\text {eff }} \approx 600 \mathrm{~K}$. Alternatively, a slightly lower metallicity would also fit, but B10 excluded an L subdwarf classification of object A, and the kinematics of the system is clearly not typical of the Galactic halo or thick disc. The blue colour of object A could also be caused by high surface gravity as discussed by Burgasser et al. (2008) for the class of blue L dwarfs. If the high gravity is correct, then the evolution models of Saumon \& Marley (2008, their Fig. 4) show that the system is likely $\approx 5$ Gyr old, and object $\mathrm{B}$ could have a mass of $\approx 30$ Jupiters. Further investigation will show whether objects $\mathrm{A}$ and $\mathrm{B}$ represent a wide

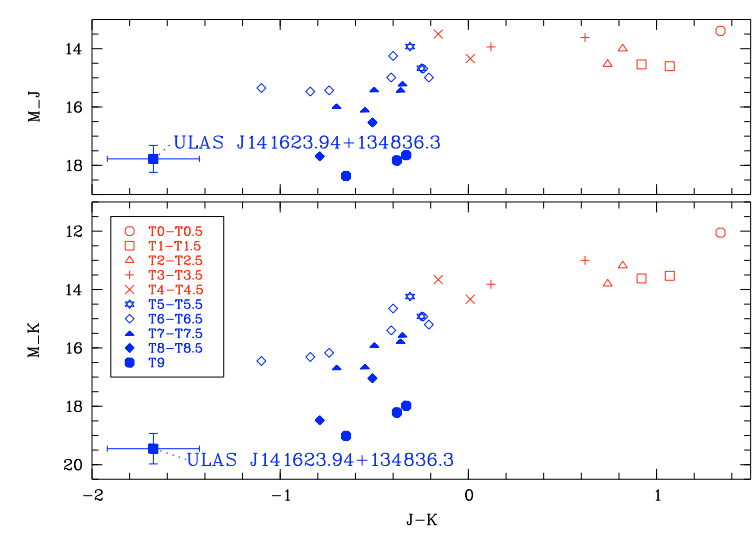

Fig. 7. Absolute magnitudes $M_{K}$ (bottom) and $M_{J}$ (top) vs. $J-$ $K$ colour (in MKO system) for all $\mathrm{T}$ dwarfs with measured trigonometric parallaxes as listed in Leggett et al. (2010) and for ULAS J141623.94+134836.3 (object B) with error bars obtained from using the parallax of object $\mathrm{A}$ (solution 4-A) and the magnitude errors of object B (Table 1).

binary brown dwarf or a much older analogue of the young lowmass star plus massive planet system 2MASS 1207-3932AB (Gizis 2002; Chauvin et al. 2004).

Acknowledgements. Data from the UKIDSS 6th data release, the SDSS DR7, the 2MASS, and the SSS were used for this work. We thank Natasha Maddox for helpful advice on UKIDSS data and Hans Zinnecker for reading a first version of the manuscript. We thank the referee, Dr. Sandy Leggett, for her prompt report, which helped us to improve the paper.

\section{References}

Abazajian, K. N., Adelman-McCarthy, J. K., Agüeros, M. A., et al. 2009, ApJS, 182,54

Bowler, B. P., Liu, M. C., \& Dupuy, T. J. 2010, ApJ, 710, 45 (B10)

Burgasser, A. J., Kirkpatrick, J. D., Brown, M. E., et al. 2002, ApJ, 564, 421

Burgasser, A. J., Looper, D. L., Kirkpatrick, J. D., Cruz, K. L., \& Swift, B. J. 2008, ApJ, 674, 451

Burningham, B., Pinfield, D. J., Leggett, S. K., et al. 2008, MNRAS, 391, 320 Burningham, B., Pinfield, D. J., Leggett, S. K., et al. 2009, MNRAS, 395, 1237 Casali, M., Adamson, A., Alves de Oliveira, C., et al. 2007, A\&A, 467, 777

Chauvin, G., Lagrange, A.-M., Dumas, C., et al. 2004, A\&A, 425, L29

Delorme, P., Delfosse, X., Albert, L., et al. 2008a, A\&A, 482, 961

Delorme, P., Willott, C. J., Forveille, T., et al. 2008b, A\&A, 484, 469

Dye, S., Warren, S. J., Hambly, N. C., et al. 2006, MNRAS, 372, 1227

Gelino, C. R., Kirkpatrick, J. D., \& Burgasser, A. J. 2009, online database for $752 \mathrm{~L}$ and T dwarfs at DwarfArchives.org (status: 16 November, 2009)

Gizis, J. E. 2002, ApJ, 575, 484

Gudehus, D. H. 2001, BAAS, 33, 850

Hambly, N. C., MacGillivray, H. T., Read, M. A., et al. 2001, MNRAS, 326, 1279

Hambly, N. C., Collins, R. S., Cross, N. J. G., et al. 2008, MNRAS, 384, 637

Harrington, R. S., \& Dahn, C. C. 1980, AJ, 85, 454

Hewett, P. C., Warren, S. J., Leggett, S. K., \& Hodgkin, S. T. 2006, MNRAS, 367,454

Lawrence, A., Warren, S. J., Almaini, O., et al. 2007, MNRAS, 379, 1599

Leggett, S. K., Burningham, B., Saumon, D., et al. 2010, ApJ, 710, 1627

Looper, D. L., Kirkpatrick, J. D., \& Burgasser, A. J. 2007, AJ, 134, 1162

Mainzer, A. K., Kirkpatrick, D., Wright, E., Padgett, D., \& McLean, I. 2009, BAAS, 41,365

Saumon, D., \& Marley, M. S. 2008, ApJ, 689, 1327

Schmidt, S. J., West, A. A., Burgasser, A. J., Bochanski, J. J., \& Hawley, S. L. 2010, AJ, 139, 1045 (S10)

Skrutskie, M. F., Cutri, R. M., Stiening, R., et al. 2006, AJ, 131, 1163

Smart, R. L., Jones, H. R. A., Lattanzi, M. G., et al. 2009 [arXiv: 0912 . 3163]

Tinney, C. G., Burgasser, A. J., Kirkpatrick, J. D., \& McElwain, M. W. 2005, AJ, 130,2326

Tokunaga, A. T., Simons, D. A., \& Vacca, W. D. 2002, PASP, 114, 180

Warren, S. J., Mortlock, D. J., Leggett, S. K., et al. 2007, MNRAS, 381, 1400

Wright, E. L. 2008, EAS Publ. Ser., 33, 57 